\title{
OAM Generation in Optical Fibre and Free Space Devices
}

\author{
Martynas Beresna, ${ }^{1,}$, Shankar Pidishety ${ }^{1,2}$, Mohammed Imran Mustafa Abdul Khudus ${ }^{1}$, Rand \\ Ismaeel', Yun Wang ', Patrick Gregg ${ }^{3}$, Siddharth Ramachandran ${ }^{3}$, Balaji Srinivasan ${ }^{2}$, Gilberto \\ Brambilla ${ }^{l}$
}

${ }^{1}$ Optoelectronics Research Centre, University of Southampton, Southampton, SO17 1BJ, U.K. ${ }^{2}$ Indian Institute of Technology Madras, Chennai-600036, India

${ }^{3}$ Electrical and Computer Engineering Department, Boston University, 8 St Mary’s St, Boston, USA

Email: M.Beresna@soton.ac.uk

\begin{abstract}
Orbital angular momentum (OAM) beam generation has been investigated using all-fibre and free space configurations. In the first approach, the composite fused coupler is based on a single mode fibre (SMF) and an air-core fibre. The second approach exploits geometrical phase introduced by nanostructuring of silica glass. Both approaches are demonstrated to achieve power coupling efficiencies in excess of $80 \%$ at telecom wavelengths.
\end{abstract}

Keywords: OAM modes, fibre couplers, nanostructured glass

\section{INTRODUCTION}

Orbital angular momentum (OAM) beams have the unique feature of a helical phase front and have attracted much attention because of the potential applications in telecom, machining and optical manipulation [1]. OAM generation has represented a great challenge as it is often associated to complex set-ups that are lossy and cannot be multiplexed. Here we present two approaches that have been used in the generation of OAM modes in all fiberized components and space variant half wave plates.

\section{OPTICAL FIBRE COUPLER}

Composite fused optical fibre couplers have been used to excite specific higher order modes (HOMs) in few mode fibres [2]. Here the few-mode fibre is replaced with an air-core fibre, thus allowing for the selectively excitation of OAM modes by launching light in the singlemode fibre (SMF) pigtail. The SMF used in these experiments was a Corning SMF28e with cut-off wavelength at $\lambda=1.25 \mu \mathrm{m}$, while the OAM air-core fibre was designed at Boston University and its refractive index profile is shown in Fig. 1. By removing the modal degeneracy and quashing intermodal cross talk, the OAM mode fibre supports the stable propagation of the OAM modes [3] In the composite coupler, the two fibres are pre-tapered in such a way that the modes propagating in the two waveguides have the same propagation constant, i.e. the same effective index $n_{\text {eff }}[2]$.

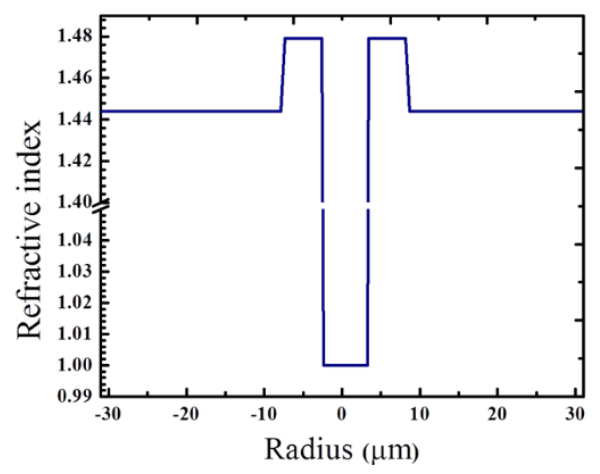

Fig. 1 Refractive index profile of the air-core fibre used in the experiments.

The phase matching condition for different degrees of tapering of the two fibres was investigated using COMSOL Multiphysics ${ }^{\circledR}$ eigen mode solver. Simulations showed that the SMF had to be pre-tapered to $\sim 75.8 \mu \mathrm{m}$. The pretapered SMF was aligned with the OAM fibre and the coupler was manufactured using the modified flame brushing technique at $\sim 1400^{\circ} \mathrm{C}$ using a ceramic micro heater (NTT-AT, Japan) [4]. Pulling ended when the power exiting the OAM fibre was maximized. When phase matching is achieved, the superposition of excited OAM modes is fine tuned by controlling the input polarization, with each circular polarization exciting one specific OAM mode.

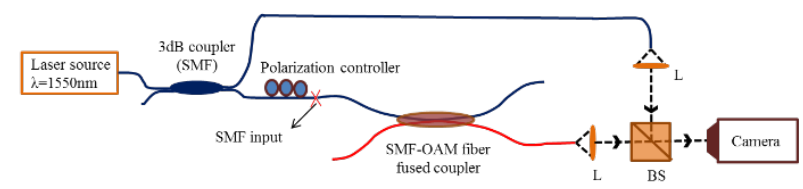

Fig. 2 Schematic of the set-up used to characterize the coupler output through the air-core fibre. L-collimating lens, BS-free space beam splitter

The output of the OAM fibre was characterized using the set-up shown in Fig. 2: light from a $\lambda=1550 \mathrm{~nm}$ laser 
source is split into two arms using a $3 \mathrm{~dB}$ coupler: while one of the coupler ports is spliced to a polarization controller and to the fabricated OAM coupler, the other port is used as SMF-based reference arm in the interference setup. The output modes from the air-core OAM fibre and from the reference SMF are interfered using a free space beam splitter. The field pattern is then imaged using a CCD camera (MicronViewer-7290A), as shown in Fig. 3.
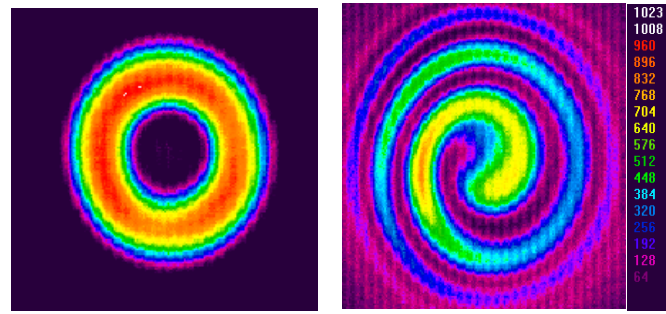

Fig. 3 (left) Far field image of the OAM beam. (right) Spiral interference patterns resulting from the interference between the OAM and the Gaussian reference beams.

Fig. 3 (left) shows the doughnut intensity distribution at the air-core fiber output when the reference arm is blocked. A polarization measurement performed using a polarizer and quarter wave plate revealed that this beam is circularly polarized. Fig. 3(right) shows the intensity pattern recorded when the reference arm was aligned with that from the air-core fiber, exhibiting the characteristic spiral interference commonly observed when OAM modes interfere with Gaussian beams. The overall coupling efficiency of the composite coupler was estimated to be of the order of $\sim 80 \%$

\section{SPACE VARIANT HALF WAVE PLATE}

An alternative approach for exciting OAM beams is by exploiting geometrical phase. The wavefront is modified by introducing spatially varying anisotropy and is a result of Pancharatnam-Berry phase [5]. Currently, this approach is widely explored for demonstrating extremely thin optical elements. Recently, this principle was implemented by nanostructuring silica glass with an ultrafast laser source. The laser induced features are smaller than the wavelength of light and thus exhibits strong form birefringence. The ability to control orientation of nanostructures enables printing arbitrary phase patterns. One of the first elements demonstrated using this technique was OAM mode converters designed for circular polarization [6] (Fig. 4). The conversion efficiency of these optical elements is limited by optical scattering, which has predominantly Rayleigh dependence. As a result, insertion losses are less than $20 \%$ at telecom wavelengths. Another important feature is high damage threshold of these devices, which allows to exploit them for high laser power applications. As fabrication technique is a direct writing method, it allows implementing optical elements as small as tens of microns. Thus, it can potentially can be integrated into the core of an optical fibre. Recently OAM modes with charge as high as 100 were successfully generated using this approach demonstrating viability of this approach for high order OAM modes [7].
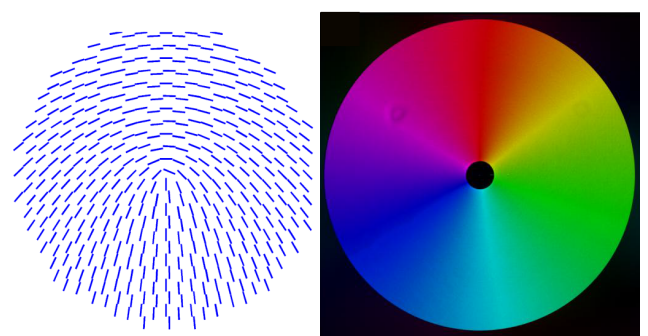

Fig. 4 (left) Schematic drawing of nanogratings distribution in a OAM mode converter. The blue dashes indicate the direction of the nanopattern. (right) A fabricated mode converter. The brightness represents the retardance magnitude while the colour indicates the direction of the slow axis

Important feature of these mode converters is sensitivity of the handedness of circular polarization. Essentially for any state of polarization the mode converter will produce two modes with opposite charge and handedness of circular polarization. The total angular momentum carried by the beam will depend on the ratio of two circular polarizations. This allows obtaining high purity OAM modes after converting a beam into two orthogonal linear polarizations and separating them with a polarizing beam splitter. Experimentally the contrast of OAM mode was improved from $3 \%$ (after the mode converter) to $>0.1 \%$ by additional polarization filtering. Additionally, this polarization sensitivity was exploited for controlling angular momentum carried by the laser beam without affecting the intensity distribution, which is a useful function for optical trapping of small particles and studying of their interaction with surfaces [8]. Total angular momentum was controlled by adjusting the ratio of two circular polarizations with a quarter-wave plate [8]. As a result, tightly focused beam induced trapping potential for small silica beads suspended in water. The variation of OAM transferred into continuous transition from the maximum rotation speed to complete halt of silica beads.

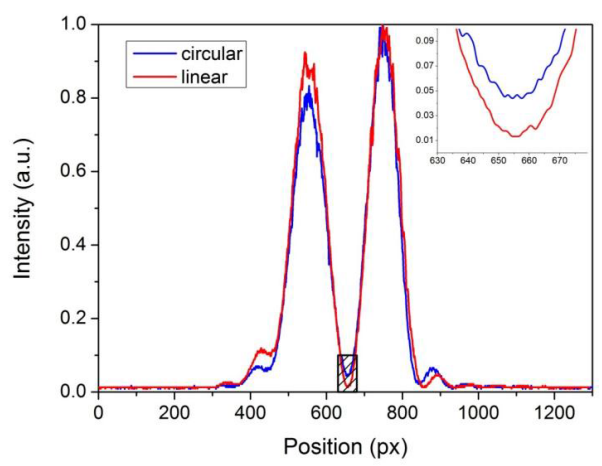

Fig. 5 OAM mode contrast after additional filtering (measurements are limited by the dynamic range of the camera). Inset: zoom of the central part. 


\section{ACKNOWLEDGMENTS}

The authors gratefully acknowledge EU funding through the IRSES project.

\section{REFERENCES}

[1] A.M. Yao, M.J. Padgett, "Orbital angular momentum: origins, behavior and applications," Adv. Opt. Photonics. 3, pp. 161-204, 2011.

[2] R. Ismaeel, G. Brambilla, "Removing the Directional Degeneracy of $\mathrm{LP}_{11}$ Mode in a Fused-Type Mode Selective Coupler" IEEE J. Lightwave Tech. 34(4), pp. 1242-1246, 2016.

[3] P. Gregg, P. Kristensen, S. Ramachandran, "Conservation of orbital angular momentum in air-core optical fibers," Optica. 2, pp. 267-270, 2015
[4] G. Brambilla, F. Koizumi, X. Feng, D.J. Richardson "Compound-glass optical nanowires," Electron. Lett. 41(7), pp.400-402, 2005.

[5] N. Yu, F. Cappasso "Flat optics with designer metasurfaces," Nat. Mater. 13, pp. 139-150, 2014.

[6] M. Beresna, M. Gecevičius, P. G. Kazansky, T. Gertus, "Radially polarized optical vortex converter created by femtosecond laser nanostructuring of glass," Appl. Phys. Lett. 98, 201101, 2011.

[7] R. Drevinskas, M. Gecevičius, M. Beresna, P. G. Kazansky, "High-topological charge vortex tweezers with continuous control of orbital angular momentum by ultrafast laser machining," Conference on Lasers and Electro-Optics, San Jose, CA, 2015, STu1L.2

[8] M. Gecevičius, R. Drevinskas, M. Beresna, P. G. Kazansky, "Single beam optical vortex tweezers with tunable orbital angular momentum," Appl. Phys. Lett. 104, 231110, 2014. 